\title{
Comunicação
}

[Communication]

\section{Diagnóstico da otite média em cães: comparação entre radiografia convencional, canalografia e audiometria de impedância}

\author{
[Diagnosis of otitis media in dogs: comparison between conventional radiography, \\ canalography and impedance audiometry] \\ C.A.L. Leite ${ }^{1}$, P.T.C. Guimarães ${ }^{2}$ \\ ${ }^{1}$ Universidade Federal de Lavras \\ Campus Universitário \\ 37200-000 - Lavras, MG \\ ${ }^{2}$ Faculdade de Medicina Veterinária e Zootecnia - Universidade Estadual Paulista Júlio de Mesquita Filho \\ Campus Universitário - Botucatu, SP
}

A otite média (OM) é uma afecção relativamente comum em medicina veterinária de cães (Leite, 2002), gerando prejuízos para o proprietário.

A radiologia do sistema vestibulococlear (SVC) constitui um dos métodos mais confiáveis e baratos no diagnóstico da OM, contudo existem casos agudos ou não reativos fornecendo resultados negativos falsos (Leite, 2002). De grande utilização em medicina humana, a audiometria de impedância, ou impedanciometria, é um método subsidiário objetivo de diagnóstico que visa estabelecer a integridade da membrana timpânica e das demais estruturas do ouvido médio (Sims, 1993; Souza et al., 2008), constituindo um instrumento de estudo confiável no diagnóstico da OM, mesmo na presença de avaliações radiográficas negativas para a doença. Logo, a busca de uma comparação entre estudos radiográficos e impedanciométricos auditivos poderá estimar o quão confiável são os primeiros exames, ao mesmo tempo em que institui, de forma pioneira, a prática audiométrica na clínica médica veterinária do país.

Foram avaliadas duas populações, uma constituída por 12 cães hígidos (grupo-controle) e outra formada por 12 animais otopatas (grupo-teste). Cada animal passou por exposições radiográficas, focando o sistema vestibulococlear. Inicialmente foi realizada radiografia convencional, com parâmetros de irradiação adequados para cada animal (Leite, 2002; Garosi et al., 2003) e em posicionamentos oblíquos ventrodorsal (esquerdo e direito) e rostrocaudal de boca aberta.

Terminada a etapa radiográfica convencional, cada cão passou por exame impedanciométrico, para avaliar cada ouvido, separadamente, e registrar os dados obtidos em software específico. Foram realizados timpanometria, mensuração da complacência estática, teste do reflexo estapedial, ipsilateral e contralateral, e determinação do volume aéreo do conduto auditivo. O impedanciômetro utilizado (EYMASA, INMISET-M ${ }^{\circledR}$ ) executou as funções de timpanometria padronizada e reflexo estapediano, trabalhando na frequência de $220 \mathrm{~Hz}$ e nível de estímulo de $85 \mathrm{~dB}$. As faixas de variação foram: sistema de pressão pneumática de -400 a $+200 \mathrm{DaPa}$ (unidade deca-Pascal); complacência de 0,2 a $5 \mathrm{~mL}$; e estímulo de 50 a 110dB HL. O sistema foi acoplado a um microcomputador, e os dados processados e gravados em mídia eletrônica. A análise qualitativa dos dados baseou-se em trabalhos de Niemeyer e Sesterhenn (1974) e Souza et al. (2008).

Imediatamente após a conclusão do procedimento anterior, e com o auxílio de seringas descartáveis estéreis de $10 \mathrm{~mL}$, o paciente recebeu de 1 a $3 \mathrm{~mL}$ de contraste

Recebido em 19 de maio de 2011

Aceito em 2 de agosto de 2011

E-mail: caca@dmv.ufla.br 
positivo iohexol $\left(\right.$ Omnipaque ${ }^{\circledR}$ ) nos condutos auditivos, que foram imediatamente obstruídos com tampões de algodão para evitar refluxo dos contrastes. Finda esta etapa, o paciente foi posicionado para nova exposição radiográfica. Para essa etapa, conhecida como radiografia contrastada positiva - canalografia,$- \quad$ o posicionamento adotado foi dorsoventral, que permitiu melhor visibilização de ambos os condutos auditivos e da própria bula timpânica, como sugerido por Trower et al. (1998). As radiografias, tanto convencionais como contrastadas, foram analisadas em busca de sinais compatíveis com otopatia média, como preconizado por Trower et al. (1998), Gotthelf (2000) e Leite (2002).

Ao se analisarem os dados radiológicos visando à detecção da OM, pôde-se inferir que tanto o método convencional quanto o contrastado positivo apresentaram falhas na detecção de pacientes confirmadamente otopatas (Tab. 1), opondo-se, parcialmente, às indicações de Trower et al. (1998) e Gotthelf (2000).

A radiografia convencional de pacientes com OM resultou em sete animais $(58,3 \%)$ com resultados negativos falsos. Também a canalografia não apontou todos os pacientes com $\mathrm{OM}$, aproximando-se da técnica anterior em cinco cães $(41,7 \%)$ da população otopata. Como lembrado por Leite (2002), a avaliação radiológica é um método falho quando utilizada isoladamente no diagnóstico da OM.

Estes resultados são preocupantes na rotina veterinária, pois a radiologia é um procedimento de diagnóstico trivial em otologia e quase sempre requisitado quando o paciente está sob suspeita de OM. Essas falhas podem ocorrer por diversas razões, como menor grau de agressividade da otite, menor tempo de cronicidade - não permite que lesões ósseas características se desenvolvam -, distribuição irregular do contraste positivo por alterações anatômicas ou presença de exsudatos intraluminais fortemente lipidogênicos - e falhas de posicionamento - posicionamento inadequado ou sobreposições -, como apontado por Leite (2002) e Garosi et al. (2003). Todavia, buscou-se eliminar o maior número possível de vícios como os citados. É possível que a maior causa dessas falhas possa ser atribuída ao menor tempo de curso da otopatia, o que não pode ser comprovado, pois os animais não tinham histórico clínico. Na Fig. 1, pode-se observar o canalograma de um cão com OM, em que a presença de contraste positivo intraluminal na bula direita atesta o diagnóstico final.

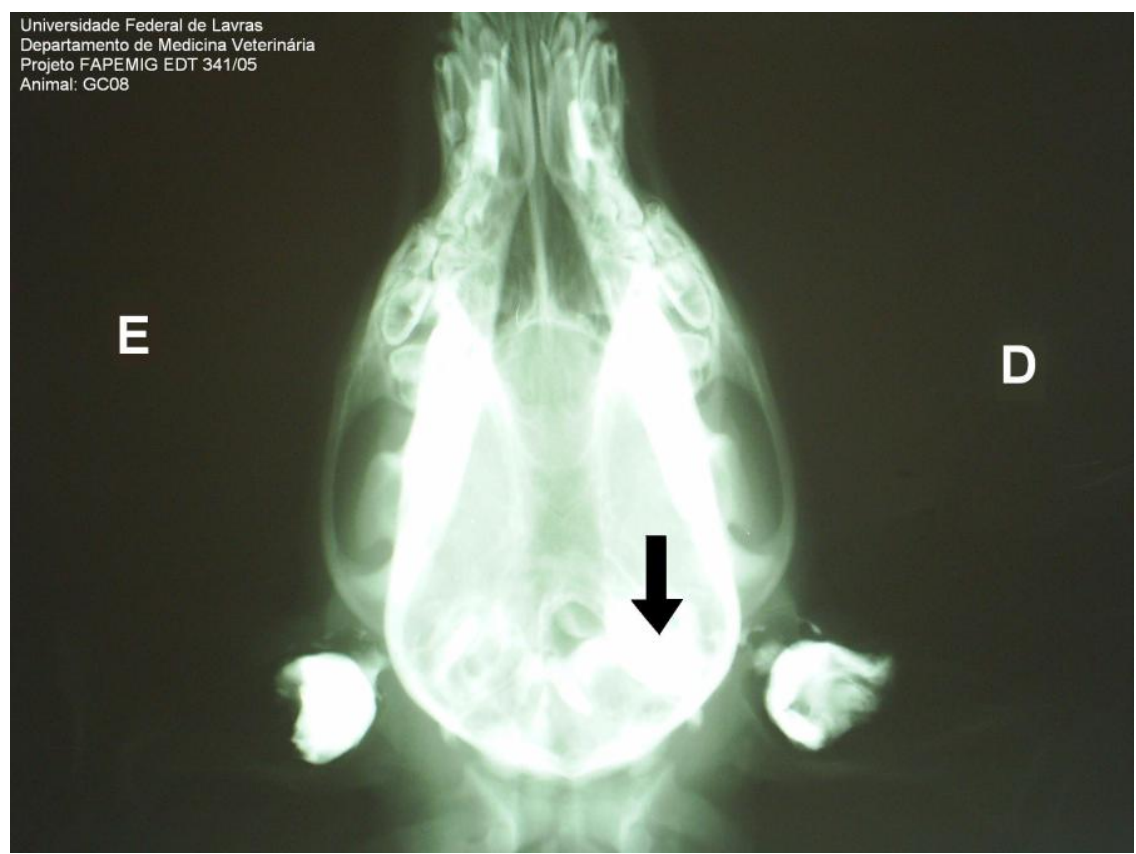

Figura 1. Radiografia contrastada positiva (canalograma) de cão com tímpano direito rompido. Observase a presença do contraste radiopaco dentro da cavidade timpânica (seta). 
A timpanometria (TP) e a medida de complacência estática (CE) confirmaram a perfuração de membranas timpânicas dos cães do grupo-teste, gerando valores abaixo de $0,12 \mathrm{~mL}$ (valor de referência, $\mathrm{VR}=0,70 \mathrm{~mL}$ ), com ausência de pico acústico em $0 \mathrm{DaPa}$ (Tab. 1). Pode-se observar no Fig. 2 que os valores de TP-CE para animais hígidos foram mais altos, média $=0,88 \mathrm{~mL}$, quando comparados com os valores de animais otopatas, média de $0,12 \mathrm{~mL}$
Sete cães $(58,3 \%)$ não demonstraram sinais compatíveis com OM ao exame radiográfico, porém com confirmação ao exame impedanciométrico, realçando o seu valor diagnóstico na avaliação da orelha média (Tab. 1), como afirmaram Souza et al. (2008). Estes dados confirmam a base do exame impedanciométrico, pois, quando a complacência das estruturas da orelha média aumenta (menor rigidez), o volume equivalente aumenta; quando há diminuição da complacência (menor rigidez), há similar redução do volume equivalente (Sims, 1993).

Tabela 1. Registros de exames auditivos físicos, radiográficos e eletrofisiológicos em cães hígidos (controle) e otopatas (teste)

\begin{tabular}{|c|c|c|c|c|c|c|c|c|}
\hline $\mathrm{N}^{\circ}$ & Grupo & RT/OT & $\mathrm{OM} / \mathrm{RX}$ & $\mathrm{OM} / \mathrm{CG}$ & TP/CE (mL) & $\begin{array}{l}\text { REI } \\
(\%)\end{array}$ & $\begin{array}{c}\text { REC } \\
(\%)\end{array}$ & $\begin{array}{l}\text { VAC } \\
\left(\mathrm{cm}^{3}\right)\end{array}$ \\
\hline 1 & Controle & - & - & - & 0,76 & 5,62 & 5,40 & 0,76 \\
\hline 2 & Controle & - & - & - & 0,96 & 5,33 & 5,39 & 0,32 \\
\hline 3 & Controle & - & - & - & 0,82 & 5,23 & 5,51 & 0,37 \\
\hline 4 & Controle & - & - & - & 0,92 & 5,44 & 5,60 & 0,29 \\
\hline 5 & Controle & - & - & - & 0,94 & 5,38 & 5,39 & 0,59 \\
\hline 6 & Controle & - & - & - & 0,82 & 5,66 & 5,44 & 0,19 \\
\hline 7 & Controle & - & - & - & 0,92 & 5,15 & 5,27 & 0,20 \\
\hline 8 & Controle & - & - & - & 0,88 & 5,49 & 5,40 & 0,31 \\
\hline 9 & Controle & - & - & - & 0,79 & 5,55 & 5,60 & 0,28 \\
\hline 10 & Controle & - & - & - & 0,93 & 5,28 & 5,32 & 0,18 \\
\hline 11 & Controle & - & - & - & 0,99 & 5,34 & 5,30 & 0,22 \\
\hline 12 & Controle & - & - & - & 0,84 & 5,62 & 5,70 & 0,60 \\
\hline 13 & Teste & + & - & + & 0,13 & 4,21 & 4,87 & 0,65 \\
\hline 14 & Teste & + & + & + & 0,17 & 5,34 & 3,65 & 0,47 \\
\hline 15 & Teste & + & + & + & 0,07 & 3,95 & 3,63 & 0,71 \\
\hline 16 & Teste & + & - & + & 0,10 & 3,87 & 3,65 & 0,59 \\
\hline 17 & Teste & + & - & - & 0,09 & 4,02 & 3,79 & 0,66 \\
\hline 18 & Teste & + & + & + & 0,09 & 4,11 & 4,08 & 0,58 \\
\hline 19 & Teste & + & + & + & 0,10 & 3,77 & 5,46 & 0,69 \\
\hline 20 & Teste & + & - & - & 0,20 & 4,00 & 4,17 & 0,70 \\
\hline 21 & Teste & + & - & - & 0,08 & 3,93 & 3,55 & 0,70 \\
\hline 22 & Teste & + & - & - & 0,14 & 3,09 & 3,26 & 0,57 \\
\hline 23 & Teste & + & + & + & 0,15 & 4,15 & 3,96 & 0,58 \\
\hline 24 & Teste & + & - & - & 0,09 & 3,77 & 3,90 & 0,69 \\
\hline
\end{tabular}

RT/OT = Ruptura timpânica por otoscopia indireta; OM/RX = Otite média em radiografia convencional; OM/CG = Otite média em canalografia; TP/CE $=$ Timpanometria/Complacência estática (referência $=0,70 \mathrm{~mL}$ ); REI $=$ Reflexo estapediano ipsilateral (referência $=5,08-5,74 \%$ a $1 \mathrm{kHz}$ ); REC $=$ Reflexo estapediano contralateral (referência $=$ $5,08-5,74 \%$ a $1 \mathrm{kHz}) ; \mathrm{VAC}=$ Volume aéreo do conduto auditivo $\left(\right.$ referência $\left.=0,4 \mathrm{~cm}^{3}\right)$. 


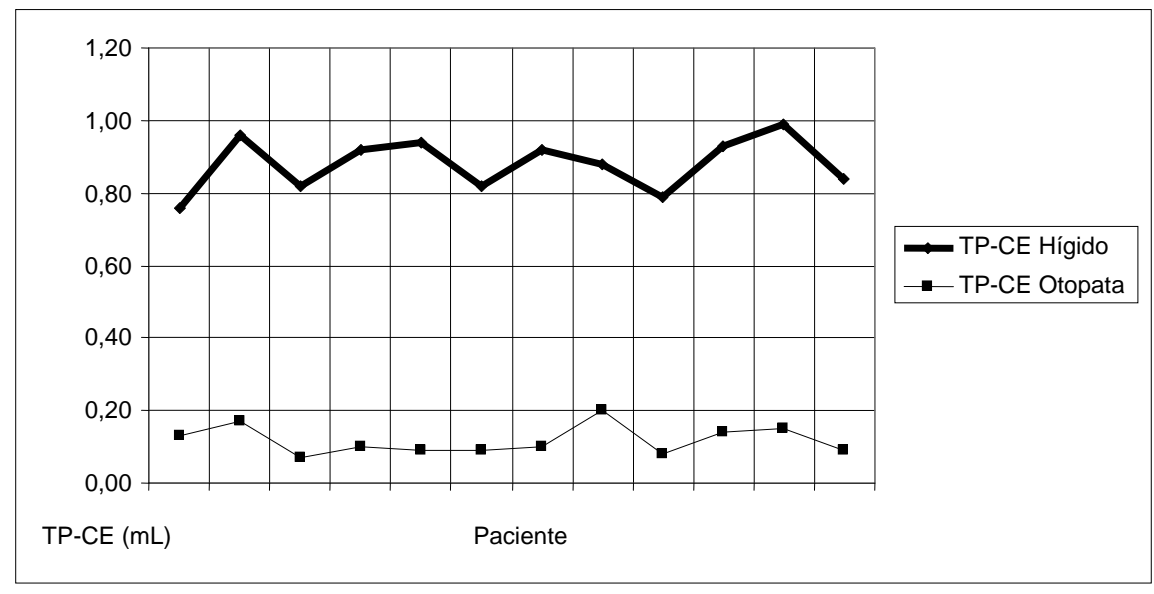

Figura 2. Timpanometria (TP) e complacência estática (CE) de cães hígidos (controle) e otopatas (teste).

O reflexo estapediano (RE) - tanto ipsilateral como contralateral - dos cães do grupo-teste demonstrou um período de declínio acústico durante o momento de limiar - sempre na faixa de $220 \mathrm{~Hz}$ e $85 \mathrm{~dB}$-, atestando claramente disfunções no nervo vestibulococlear e confirmando pesquisas realizadas por Niemeyer e Sesterhenn (1974). Dois ouvidos de pacientes diferentes - cães 14 e 19 do grupo-teste - não apresentaram declínio nessa avaliação, o que pode ser explicado pela possível integridade parcial do músculo estapédio unilateral.

Na Fig. 3, observa-se que o RE ipsilateral dos animais otopatas apresentou declínio médio de $4,0 \%$ (VR=5,1-5,7\%), enquanto o RE ipsilateral para seus pares hígidos manteve-se na faixa de $5,4 \%$. Da mesma forma, a média do RE contralateral em animais otopatas, $4,0 \%$, foi mais baixa que a verificada em seus congêneres não otopatas, $5,44 \%$, o que pode ser visibilizado na Fig. 4. A interpretação dos resultados nos animais do grupo-teste pode sugerir a presença de disacusias do tipo neurossensorial, especialmente aquelas que ocorrem por lesões vestibulares devido à OM (Sims, 1993; Souza et al., 2008).

Os animais do grupo-controle apresentaram volume aéreo do conduto auditivo (VCA) em torno de $0,36 \mathrm{~cm}^{3} \quad\left(\mathrm{VR}=0,4 \mathrm{~cm}^{3}\right)$. Já nos cães otopatas, houve aumento acentuado desse volume (média $=0,63 \mathrm{~cm}^{3}$ ), atestando a presença de ruptura timpânica (Fig. 5), como sugerido por Souza et al. (2008). Este aumento certamente ocorreu em razão do maior espaço aéreo gerado entre o meato auditivo externo e a parede óssea medial da bula, apesar dos variados graus de estenose do conduto auditivo e presença de efusão intraluminal. Três animais (1, 5 e 12) apresentaram valores de VCA estatisticamente diferentes dos seus pares (Tab. 1), o que pode ser explicado pela grande diversidade de alterações anatômicas que podem influenciar os resultados desse exame. Os cães hígidos podem ter alterações próprias do conduto auditivo, fazendo com que o maior calibre do conduto origine valores mais altos que os considerados de referência para a espécie. É oportuno lembrar que em cães há alta variabilidade anatômica do conduto auditivo, o que pode influenciar na interpretação final desse teste.

A presença de efusão timpânica intraluminal foi detectada tanto na radiologia convencional como nos testes eletrofisiológicos auditivos. $\mathrm{Na}$ análise radiográfica, foram sinalizados cinco cães $(41,7 \%)$ com efusão, enquanto a audiometria de impedância apontou oito animais (66,7\%) apresentando aumento de líquidos no ouvido médio. Dessa maneira, a impedanciometria mostrou-se bem mais sensível à detecção desse tipo de alteração, possivelmente devido à maior sensibilidade para pequenas coleções líquidas e à diferença de impedância que existe entre os meios próprios do sistema vestibulococlear. Assim, pode-se afirmar que a impedanciometria auditiva é válida no exame diagnóstico da OM de cães, por apresentar maior confiabilidade quando comparada aos métodos radiológicos usuais. 


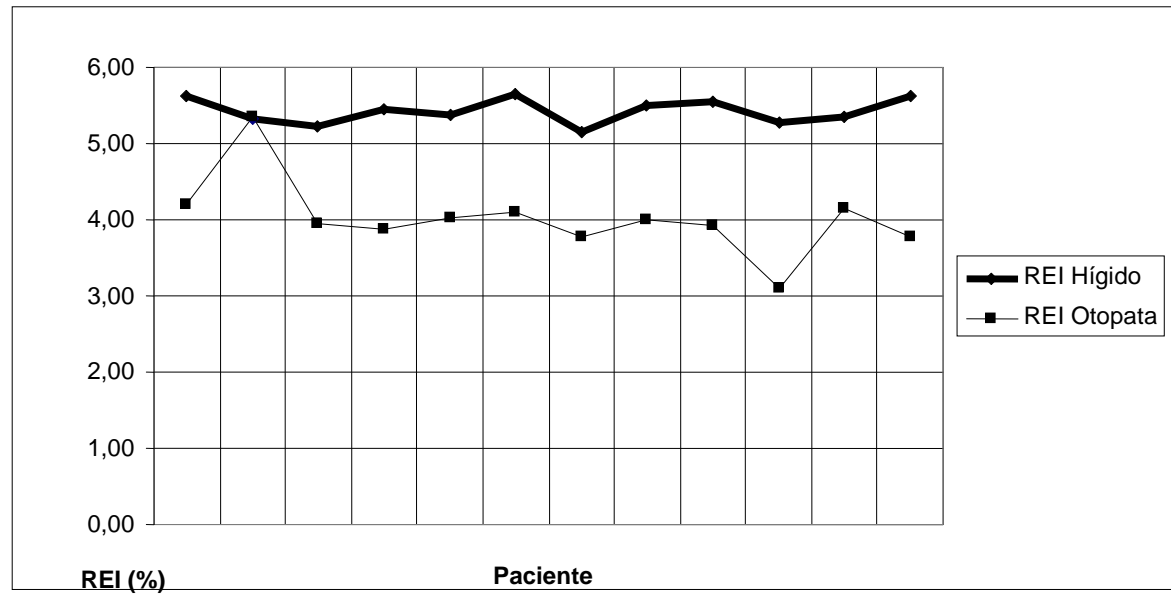

Figura 3. Reflexo estapediano ipsilateral (REI) de cães hígidos (controle) e otopatas (teste).

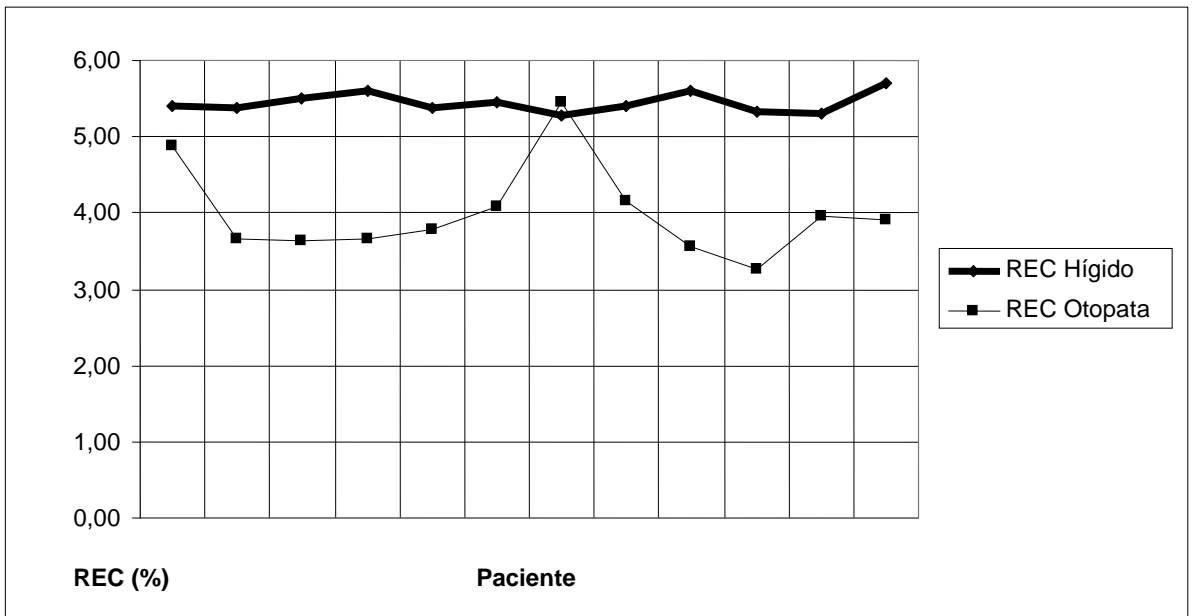

Figura 4. Reflexo estapediano contralateral (REC) de cães hígidos (controle) e otopatas (teste).

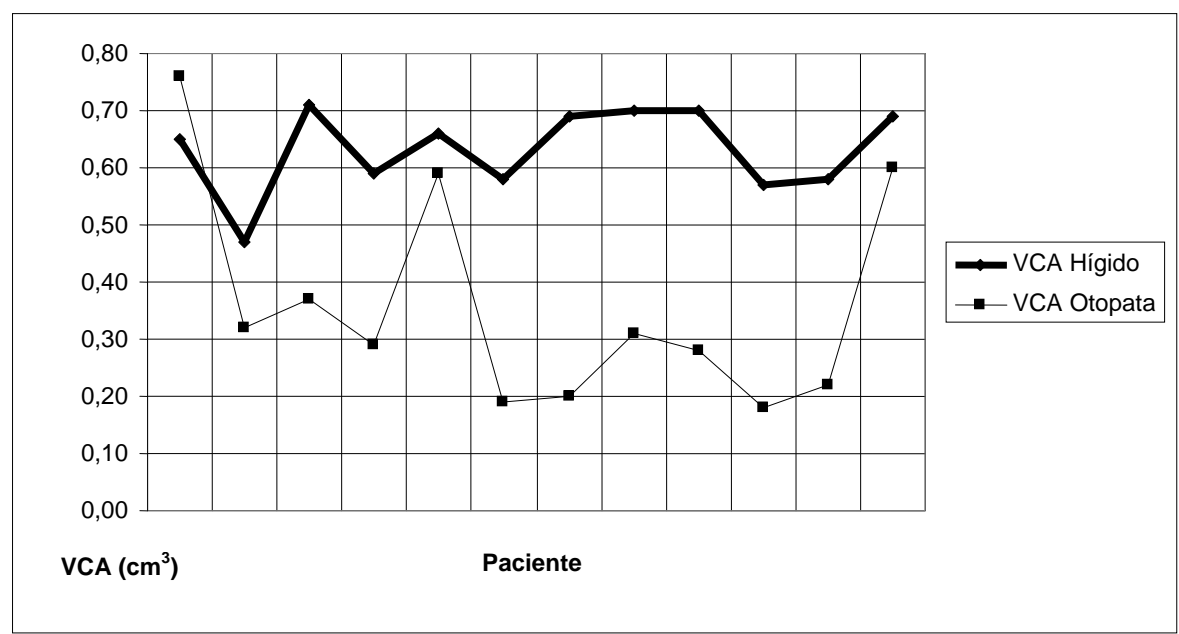

Figura 5. Volume aéreo do conduto auditivo (VCA) de cães hígidos (controle) e otopatas (teste).

Palavras-chave: cão, radiologia, otoscopia, impedanciometria, otite, orelha 


\begin{abstract}
Radiography (conventional and contrasted - canalography) and audioelectrophysiologic (impedance audiometry) tests were correlated with otitis media in 12 middle ear affected and 12 healthy dogs. When comparing both diagnostic methods, it was found that the reliability in the detection of otitis media in dogs was $100 \%$ for the first and 58.3-60.0\% for the latter. Thus, it was possible to attest that the impedance audiometry was the most accurate method in the identification of otitis media in dogs when compared with radiographic assessments.
\end{abstract}

Keywords: dog, radiology, otoscopy, impedance audiometry, otitis, ear

\section{AGRADECIMENTO}

O pesquisador agradece à Fapemig, pela concessão de auxílio-pesquisa e bolsa de iniciação científica (Projeto EDT-341-05).

\section{REFERÊNCIAS BIBLIOGRÁFICAS}

GAROSI, L.S.; DENNIS, R.; SCHWARZ, T. Review of diagnostic imaging of ear diseases in three dog and cat. Vet. Radiol. Utrasound, v.44, p.137-146, 2003.

GOTTHELF, L.N. Diagnosis of otitis media in dogs. Waltham Focus, v.10, p.24-30, 2000.

LEITE, C.A.L. A avaliação radiográfica no diagnóstico da otite média em caninos e felinos. Rev. Bras. Med. Vet., v.1, p.35-43, 2002.
NIEMEYER, W.; SESTERHENN, G. Calculating the hearing threshold from the stapedius reflex threshold for different sound stimuli. Audiology, v.13, p.421-429, 1974.

SIMS, M.H. Evaluación electrodiagnóstica de la audición. Clin. Vet. N. Am. - Pract. Clin. Peq. Anim., v.18, p.211-245, 1993.

SOUZA, L.C.A.; PIZA, M.R.T.; ALVARENGA, K.F. et al. Eletrofisiologia da audição e emissões otoacústicas. São Paulo: Tecmedd, 2008. 372p.

TROWER, N.D.; GREGORY, S.P.; RENFREW, $\mathrm{H}$. et al. Evaluation of the canine tympanic membrane by positive contrast ear canalography. Vet. Rec., v.142, p.78-81, 1998. 